\title{
Restorative Space Analysis by Jaw Motion Tracking Using a Template in Completely Edentulous Patients
}

\author{
Chol-Gwan Han, Young-Bum Park (D), June-Sung Shim ${ }^{(D)}$ and Jong-Eun Kim * \\ Department of Prosthodontics, Yonsei University College of Dentistry, Yonsei-ro 50-1, Seodaemun-gu, \\ Seoul 03722, Korea; hancg@yuhs.ac (C.-G.H.); drybpark@yuhs.ac (Y.-B.P.); jfshim@yuhs.ac (J.-S.S.) \\ * Correspondence: gomyou@yuhs.ac; Tel.: +82-2-2228-3160
}

Citation: Han, C.-G.; Park, Y.-B.; Shim, J.-S.; Kim, J.-E. Restorative Space Analysis by Jaw Motion Tracking Using a Template in Completely Edentulous Patients. Appl. Sci. 2021, 11, 3933. https:// doi.org/10.3390/app11093933

Academic Editor: Yong-Deok Kim

Received: 1 March 2021

Accepted: 25 April 2021

Published: 27 April 2021

Publisher's Note: MDPI stays neutral with regard to jurisdictional claims in published maps and institutional affiliations.

Copyright: (c) 2021 by the authors. Licensee MDPI, Basel, Switzerland. This article is an open access article distributed under the terms and conditions of the Creative Commons Attribution (CC BY) license (https:/ / creativecommons.org/licenses/by/ $4.0 /)$.

\begin{abstract}
Improvements in computer-aided design/computer-aided manufacturing technologies have led to multiple attempts being made to simplify and improve the workflow of prosthesis fabrication for completely edentulous patients. However, most attempts still involve the conventional methods of impression-making and recording the maxillomandibular relationships using alginate, rubber impression materials, and wax materials. In the case of a completely edentulous arch, the presence of movable tissues and the absence of stable landmarks make it difficult to perform direct digitization using an intraoral scanner and to digitally determine the vertical dimension. In the alternative technique described herein, data are obtained by scanning a template such as the patient's existing old dentures and jaw movement data using target materials and an optical scanner, and an appropriate maxillomandibular relationship that has the desired restorative space is determined on the basis of the obtained trajectory of mandibular movements while opening and closing the mouth. After designing dentures on the basis of the newly established maxillomandibular relationships and performing a try-in process, the final dentures can be manufactured. This alternative technique can reduce the need for multiple visits and complex procedures, improving the workflow for fabricating prostheses with the correct maxillomandibular relationships for individual patients.
\end{abstract}

Keywords: target tracking; completely edentulous patient; digital dentistry; CAD/CAM; complete removable denture; vertical dimension; restorative space analysis

\section{Introduction}

The reproduction of optimal occlusion in completely edentulous patients requires the accurate determination of the maxillomandibular relationship. The conventional technique used to record the maxillomandibular relationship in an edentulous patient involves using record bases and wax occlusal rims to determine the occlusal plane, vertical dimension, and centric relationship. Moreover, a facebow transfer is used to determine the position of the hinge axis, with the obtained information transferred to an articulator to reproduce accurate jaw movements. However, specific complexities and anatomical characteristics of jaw movements in individual patients often result in mechanical articulators failing to accurately reproduce jaw movements, with them instead possibly even introducing their own errors [1].

The ongoing improvements in computer-aided design/computer-aided manufacturing (CAD/CAM) technologies since 1980 have resulted in CAD/CAM being universally utilized in the diagnosis and treatment of dental patients [2]. However, its application has primarily been limited to the fabrication of fixed prostheses with natural tooth abutments and/or dental implants. Although the use of a digital workflow in the fabrication of removable dental prostheses for completely edentulous patients was first suggested in 1994 [3], only minor improvements were made until recently owing to the complexity of the treatment workflow for this population, which includes impression-making, determining the maxillomandibular relationship, and identifying suitable locations for the placement of 
artificial teeth on the basis of both muscular and phonetic considerations [4]. Various types of commercial workflow systems for applying digital methods to treat completely edentulous patients were introduced in the 2010s as solutions to the considerable hindrances in the digital fabrication of complete removable dentures, including the AvaDent Digital Denture (Global Dental Science, Scottsdale, AZ, USA), Dentca CAD/CAM System (Dentca, Los Angeles, CA, USA), and Baltic Denture System (Merz Dental, Lütjenburg, Germany) [5]. These methods can produce complete removable dentures with fewer patient visits [6], but they are primarily based on conventional impression-making procedures using impression materials. In addition, since the maxillomandibular relationship could not be determined digitally, the traditional method using record bases and wax occlusion rims was used before scanning the edentulous models and their relationships [7].

Various methods for tracking jaw movements utilizing mechanical, electromagnetic, and ultrasonic devices have been introduced recently [8-11]. Although several methods for transferring jaw movement data into digital software have been suggested [12], they require the patient to wear bulky equipment, which interferes with natural jaw movements and is also uncomfortable, restricting their clinical application. A target-tracking method has been described to overcome these limitations, wherein target materials and an optical scanner are used to scan jaw movements, and the data are overlaid onto a full arch model using reference points [13-16]. This approach is more rapid than conventional methods, is conducive to patient comfort and natural movements, and does not involve exposure to ionizing radiation [13-16]. However, within the limits of the author's knowledge, no technical note has yet been reported that analyzed the restoration space of a complete edentulous patient using the jaw motion tracking technique and attempted vertical dimension alteration.

The present study applied a technique in which target materials attached to the anterior teeth and an optical scanner for target tracking are used to record the trajectory of mandibular movements while opening and closing the mouth-the envelope of functionin completely edentulous patients for the evaluation of restorative space and its use in the subsequent prosthetic treatment plan.

\section{Materials and Methods}

\subsection{Attaching Target Materials to the Template and Performing Anterior Jaw Movement Tracking}

The patient selected for this technique had a completely edentulous maxillary arch and a dentulous mandibular arch. The retention and overall quality of the patient's old denture were evaluated. If the retention and/or fit between the intaglio surface of the denture and residual ridge were not adequate, border molding and relining/rebasing procedures would be used to improve the intaglio surface and retention of the denture.

For this technical note, a target in the form of a double circle was used, and it was attached to the upper and lower central incisors and canines, respectively. The selection of the shape of the target and the location of attachment were determined through the results of tracking accuracy identified through preliminary research (Figure S1). Four target materials (Target Stickers, Medit, Seoul, Korea) were attached to the central incisor and canines of the patient's denture and antagonist arch (Figure 1A), and an intraoral scanner (i500, Medit) was used to scan the maxillary and mandibular anterior teeth with the attached target materials and record the relationship between the target materials and teeth (Figure 1B). An optical scanner (Rexcan CS2, Medit) for target tracking was placed at an appropriate distance from the anterior dentition where the teeth were adequately focused, and the locations of the targets were detected after retracting the patient's cheeks using a mouth retractor. The patient was asked to open and close their mouth repeated while the optical scanner was used to record the jaw movements by tracking the movements of the targets. When a template such as dentures is placed in the oral cavity, it is necessary to evaluate whether the facial midline matches the dental midline and whether the lip support is sufficient. Those information was reflected in the subsequent design process. 

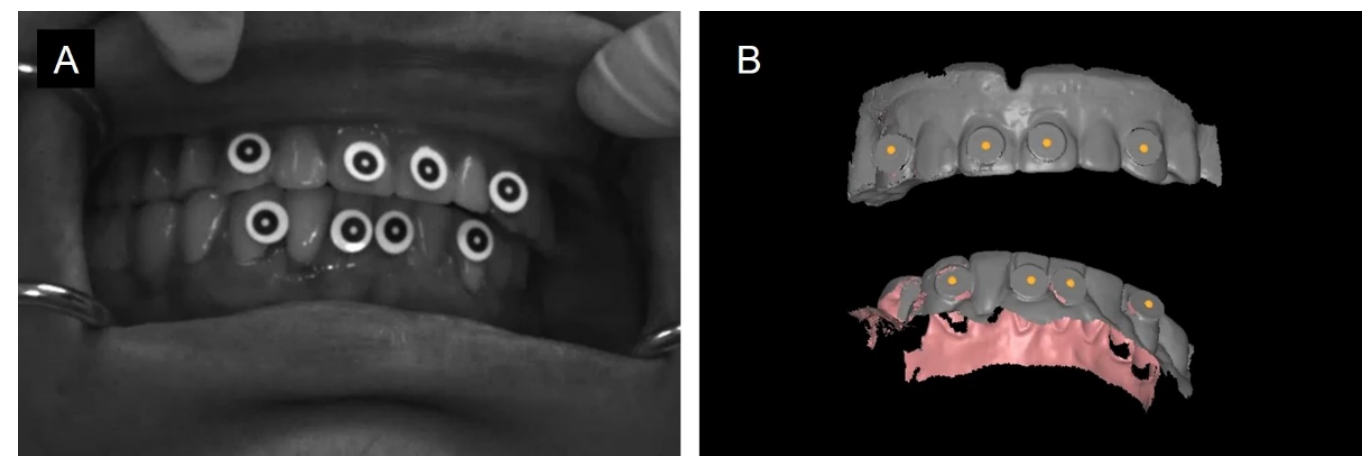

Figure 1. (A) Target materials were attached to the anterior teeth of the patient's old denture and antagonist arch. (B) Anterior regions of both arches were scanned using an intraoral scanner to detect the targets.

2.2. Implementing Appropriate Jaw Movement of Complete Edentulous Patients by Aligning with the Scanned Data of the Template

The patient's denture and antagonist arch were scanned, and the obtained data were aligned with the scan data obtained using an intraoral scanner as described above (Figure 2A). In this step, the movement of the target materials was converted into movement of the denture and antagonist arch (Figure 2B,C).
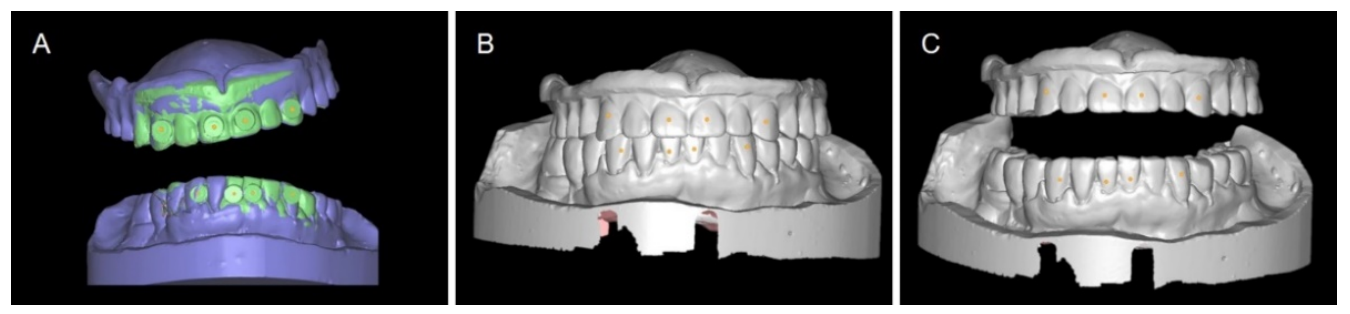

Figure 2. (A) Data scanned from all areas of the denture and plaster model were aligned. (B) The mouth was in closed state while tracking was in progress. (C) Tracking movement while opening the mouth.

The intaglio surface of the scanned denture was selected using mesh modeling software (Meshmixer, Autodesk, San Rafael, CA, USA). The "flip" function of the software (Figure 3A) was used to obtain the residual ridge data of the completely edentulous arch (Figure 3B). By selecting the negative region of the intaglio surface to be flipped and using the flip function, we were able to obtain positive mesh data in the form of residual ridges (Video S1). Since the scanned denture data and residual ridge data obtained via flipping had the location information aligned in the same coordinate system, the recorded tracking data was able to be converted into new tracking data between the residual ridge and antagonist arch during opening and closing of the mouth (Video S2).
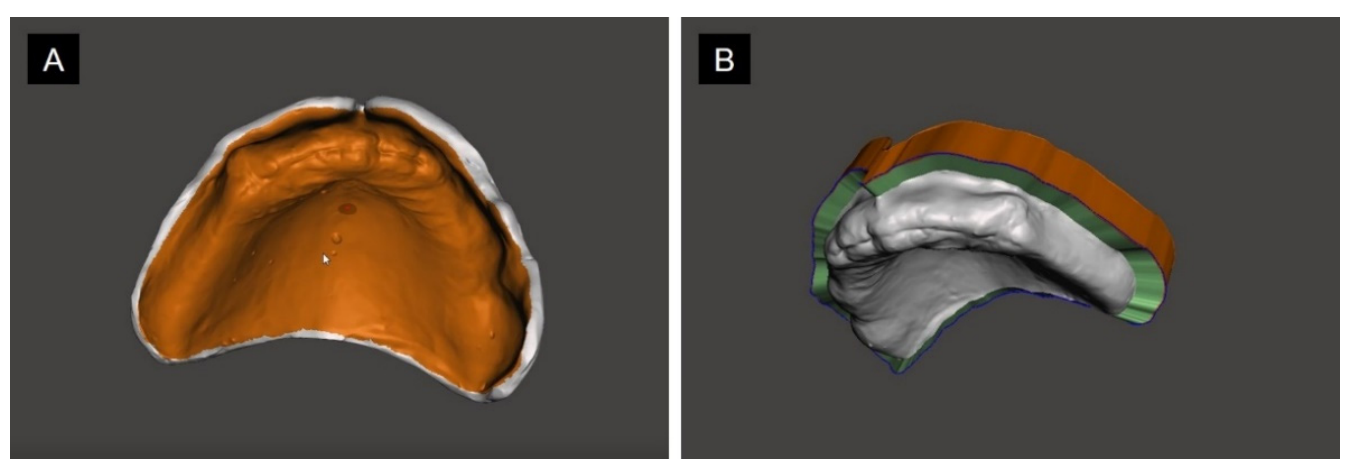

Figure 3. (A) Selecting the intaglio surface of the denture. (B) Producing a digital edentulous model using the "flip" function. 
Figure 4 shows that an old denture that is severely worn would restrict the restorable space. The vertical dimension can be altered during opening and closing of the mouth, and in the tracking trajectory, the maxillary and mandibular mesh files corresponding to the desired height can be extracted from the prototype software (Figure 4C). Multiple vertical heights can be selected according to the required increase in the restorative space (Figure 5A). If the clinician plans to fabricate an implant-retained overdenture, the presence of an adequate restorative space for incorporating attachments can be determined by designing a fixed restoration, removable restoration, or attachment structure arbitrary using the CAD software (Exocad DentalCAD, Exocad, Germany) (Figure 5B).
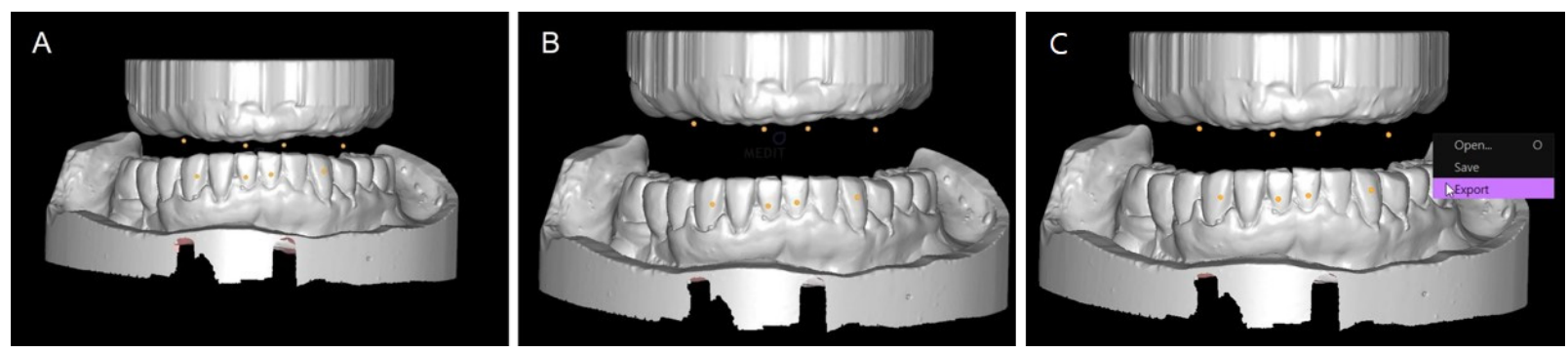

Figure 4. The jaw motion data of the old denture state implemented in Figure 2 converted into motion data in the complete edentulous arch. (A) Closed mouth. (B) Tracking movement while opening the mouth. (C) Extracting the maxillary and mandibular meshes of the desired height in the tracking trajectory.
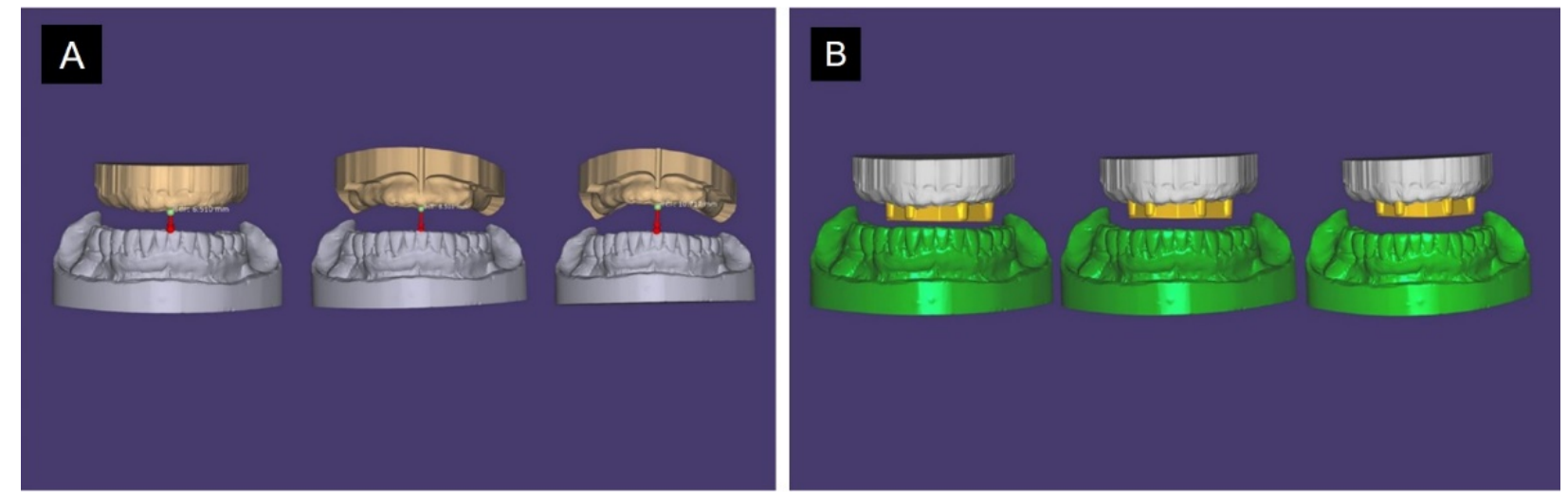

Figure 5. (A) By measuring the distance between the mandibular anterior teeth and the maxillary anterior edentulous ridge, one is able to ascertain the appropriate vertical dimension. (B) A bar attachment can be placed in the restorative space to evaluate whether it is adequate.

The maxillary and mandibular stereolithography files with the desired vertical dimension can be exported and then used to design trial dentures with the aid of the denture design module of the 3D CAD software (3Shape Dental System, 3Shape, Copenhagen, Denmark). When designing a trial denture, the clinician can adjust the anterior tooth arrangement by referring to or supplementing the lip support and aesthetics of the anterior tooth from the previous denture (Figure 6). The clinician can make trial dentures that have various designs and vertical heights. 

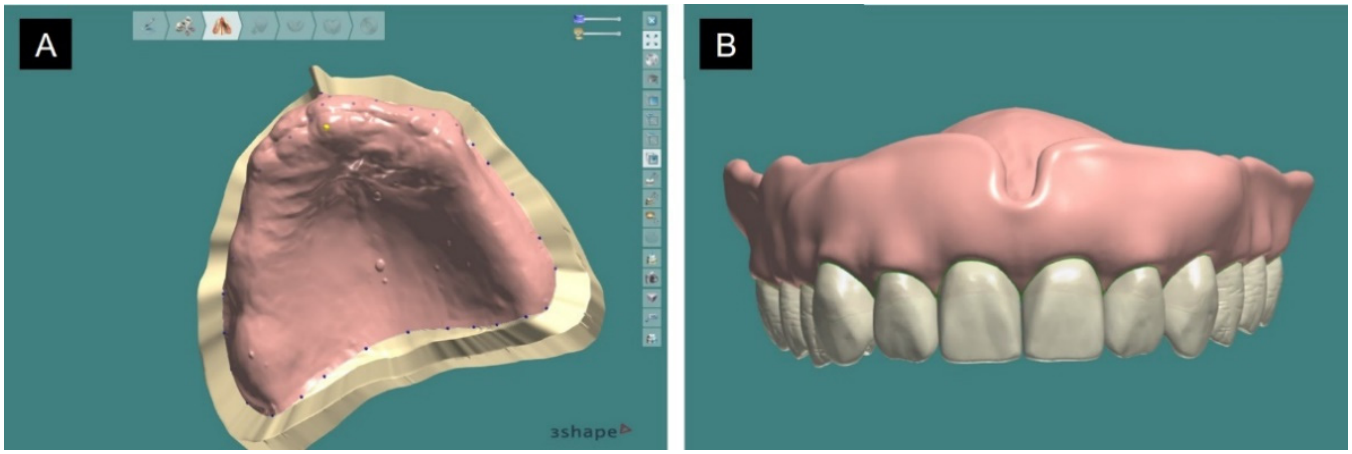

Figure 6. Trial denture designed using the denture module of the computer-aided design software with the selected vertical dimension. (A) Digital edentulous model. (B) Designed trial denture.

The designed trial dentures were printed using a 3D printer (Form3, Formlabs, Somerville, MA, USA) and 3D printing resin (NextDent C\&B, 3D Systems, Soesterberg, the Netherlands) (Figure 7A). The clinician can place each trial denture in the patient's mouth and then select the most appropriate denture for further treatment (Figure 7B). The clinician can use the trial denture to select an appropriate vertical height and restorative space, and then complete the temporary denture and provide it to the patient. For the determined vertical dimension and restorative space, the clinician can select the final prosthesis with an implant-supported fixed dental prosthesis, an implant overdenture, or a conventional fully removable denture that is consistent with that vertical dimension.
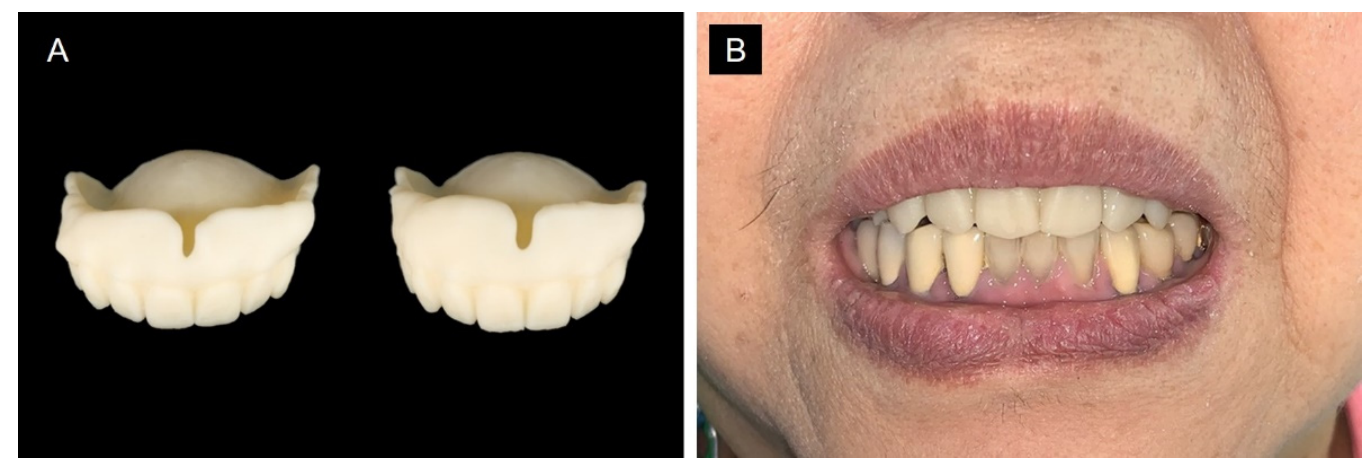

Figure 7. (A) Printed versions of the designed trial dentures. (B) The patient tried multiple trial dentures, and the one with the most appropriate vertical dimension was selected.

\section{Results}

The jaw movement tracking of a complete edentulous patient was successfully performed. By reversing the template inner surface data, we reproduced and evaluated the restorative space of the complete edentulous state during jaw movement.

\section{Discussion}

The dental technique described herein uses a digital approach to analyze the restorative space and establish a new vertical dimension using the old denture as a target-tracking template for patients whose old dentures have worn out and have a reduced vertical dimension. A previous study proposed a target-tracking method to determine maxillomandibular relationships in completely edentulous patients, but that method required the patient to wear headgear for recording the jaw relationships, and only static relationships were determined [17]. In contrast, our technique uses a target-tracking method to record the dynamic movements of the jaws and analyze the restorative space after determining the maxillomandibular relationship.

The present technique of scanning the denture using a table scanner and recording the dynamic jaw movements via a target-tracking method provides various benefits. First, 
it reduces the number of patient visits and patient discomfort associated with conventional impression-making and recording of maxillomandibular relationships. Second, the technique records the actual trajectory of mandibular movements while opening and closing the mouth, and hence the maxillomandibular relationship can be accurately ascertained in each patient. Third, since several trial dentures with different vertical dimensions can be designed and fabricated, it is possible to evaluate the restorative space for designing a solitary attachment, bar attachment, or a conventional complete removable denture. The trial denture that best suits the patient requirements can be selected, and the definitive prosthesis can be fabricated on the basis of the associated vertical dimension. Finally, the patient's old dentures are used as a template for tracking, and lip support can be modified and/or occlusion can be established on the basis of the location of teeth in the old denture. This approach can speed up the process of patient adaptation.

However, there are some limitations to this technique. First, discomfort when wearing the mouth retractor could interfere with natural jaw movements. Second, because target materials are applied to both arches, if the old denture has underextended borders and/or inappropriate fit of the intaglio surface, errors may be introduced owing to movement of the denture during jaw movements. Prescan relining of the intaglio surface of the denture or making conventional impressions to improve the fit and/or using an appropriate tracking template for the tissue surface may improve the accuracy of the workflow.

The attaching of target materials on the anterior teeth as reference points in this technique enables the reproduction of real-time mandibular movements. This makes the technique useful for determining maxillomandibular relationships in cases of complete mouth rehabilitation where the vertical dimension needs to be altered. Moreover, if optical scanners that can obtain images over a wider spatial range are used to record the 3D facial data, information about anatomical features such as the midline, interpupillary line, lip contour and margin, and Camper's line can be recorded from the patient in order to design accurate prostheses. Further research is needed to determine the accuracy of the tracking and methods for reducing the number of visits and/or chair time.

\section{Conclusions}

The technique described herein uses a target-tracking method to extrapolate dynamic jaw movements in completely edentulous patients into 3D digital data and determine the maxillomandibular relationship by evaluating the restorative space. The old denture is used as a template for target tracking. This method makes it possible to evaluate the restorative space, make a treatment plan, and fabricate a new prosthesis with accurate maxillomandibular relationships without needing to make conventional impressions or record maxillomandibular relationship using record bases or wax occlusal rims.

Supplementary Materials: The followings are available online at https:/ / www.mdpi.com/article/ 10.3390/app11093933/s1, Figure S1: Preliminary study data for evaluating tracking accuracy according to the shape and arrangement of targets. The static model mounted on the articulator was recorded using optical scanner for 7 seconds (50 frames per second, total 350 frames) to evaluate the deviation from the reference position (Mean + standard deviation). Video. S1: A video that converts the information on the inner surface of the denture into residual ridge information using the flip function, Video. S2: Converted new tracking data between the residual ridge and antagonist arch during opening and closing of the mouth.

Author Contributions: Conceptualization, C.-G.H. and J.-E.K.; methodology, J.-E.K.; software, C.G.H. and J.-E.K.; validation, C.-G.H., Y.-B.P., and J.-S.S.; formal analysis, J.-E.K.; investigation, C.-G.H.; resources, J.-S.S.; writing-original draft preparation, C.-G.H. and J.-E.K.; writing-review and editing, C.-G.H. and J.-E.K.; visualization, C.-G.H. and Y.-B.P.; supervision, J.-E.K.; project administration, J.-E.K. All authors have read and agreed to the published version of the manuscript.

Funding: This research was supported by Basic Science Research Program through the National Research Foundation of Korea (NRF) funded by the Ministry of Education (NRF-2019R1I1A1A01062792).

Institutional Review Board Statement: Not applicable. 
Informed Consent Statement: Not applicable.

Data Availability Statement: The data presented in this study are available on request from the corresponding author.

Acknowledgments: The authors thank Soobok Lee and Sungbin Im from the Medit company for their technical support.

Conflicts of Interest: The authors declare that they have no conflict of interest.

\section{References}

1. Palik, J.F.; Nelson, D.R.; White, J.T. Accuracy of an earpiece face-bow. J. Prosthet. Dent. 1985, 53, 800-804. [CrossRef]

2. Duret, F.; Blouin, J.-L.; Duret, B. CAD-CAM in dentistry. J. Am. Dent. Assoc. 1988, 117, 715-720. [CrossRef] [PubMed]

3. Maeda, Y.; Minoura, M.; Tsutsumi, S.; Okada, M.; Nokubi, T. A CAD/CAM system for removable denture. Part I: Fabrication of complete dentures. Int. J. Prosthodont. 1994, 7, 17-21. [PubMed]

4. Goodacre, C.J.; Garbacea, A.; Naylor, W.P.; Daher, T.; Marchack, C.B.; Lowry, J. CAD/CAM fabricated complete dentures: Concepts and clinical methods of obtaining required morphological data. J. Prosthet. Dent. 2012, 107, 34-46. [CrossRef]

5. Bidra, A.S.; Taylor, T.D.; Agar, J.R. Computer-aided technology for fabricating complete dentures: Systematic review of historical background, current status, and future perspectives. J. Prosthet. Dent. 2013, 109, 361-366. [CrossRef]

6. Infante, L.; Yilmaz, B.; McGlumphy, E.; Finger, I. Fabricating complete dentures with CAD/CAM technology. J. Prosthet. Dent. 2014, 111, 351-355. [CrossRef] [PubMed]

7. Bilgin, M.S.; Baytaroğlu, E.N.; Erdem, A.; Dilber, E. A review of computer-aided design/computer-aided manufacture techniques for removable denture fabrication. Eur. J. Dent. 2016, 10, 286. [CrossRef] [PubMed]

8. Flavel, S.C.; Nordstrom, M.A.; Miles, T.S. A simple and inexpensive system for monitoring jaw movements in ambulatory humans. J. Biomech. 2002, 35, 573-577. [CrossRef]

9. Soboleva, U.; Laurina, L.; Slaidina, A. Jaw tracking devices-historical review of methods development. Part I. Stomatologija 2005, 7, 67-71. [PubMed]

10. Soboleva, U.; Laurina, L.; Slaidina, A. Jaw tracking devices-historical review of methods development. Part II. Stomatologija 2005, 7, 72-76. [PubMed]

11. Wieckiewicz, M.; Zietek, M.; Nowakowska, D.; Wieckiewicz, W. Comparison of selected kinematic facebows applied to mandibular tracing. BioMed Res. Int. 2014, 2014. [CrossRef] [PubMed]

12. Solaberrieta, E.; Garmendia, A.; Minguez, R.; Brizuela, A.; Pradies, G. Virtual facebow technique. J. Prosthet. Dent. 2015, 114, 751-755. [CrossRef] [PubMed]

13. Kwon, J.H.; Im, S.; Chang, M.; Kim, J.-E.; Shim, J.-S. A digital approach to dynamic jaw tracking using a target tracking system and a structured-light three-dimensional scanner. J. Prosthodont. Res. 2019, 63, 115-119. [CrossRef] [PubMed]

14. Pinheiro, A.; Pereira, A.; Andrade, A.; Bellomo, D. Measurement of jaw motion: The proposal of a simple and accurate method. J. Med. Eng. Technol. 2011, 35, 125-133. [CrossRef]

15. Röhrle, O.; Waddell, J.N.; Foster, K.D.; Saini, H.; Pullan, A.J. Using a motion-capture system to record dynamic articulation for application in CAD/CAM software. J. Prosthodont. 2009, 18, 703-710. [CrossRef] [PubMed]

16. Zafar, H.; Eriksson, P.O.; Nordh, E.; Häggman-Henrikson, B. Wireless optoelectronic recordings of mandibular and associated head-neck movements in man: A methodological study. J. Oral Rehab. 2000, 27, 227-238. [CrossRef] [PubMed]

17. Li, W.; Xie, Q.; Wang, Y.; Sun, Y. A pilot study of digital recording of edentulous jaw relations using a handheld scanner and specially designed headgear. Sci. Rep. 2018, 8, 1-8. [CrossRef] [PubMed] 